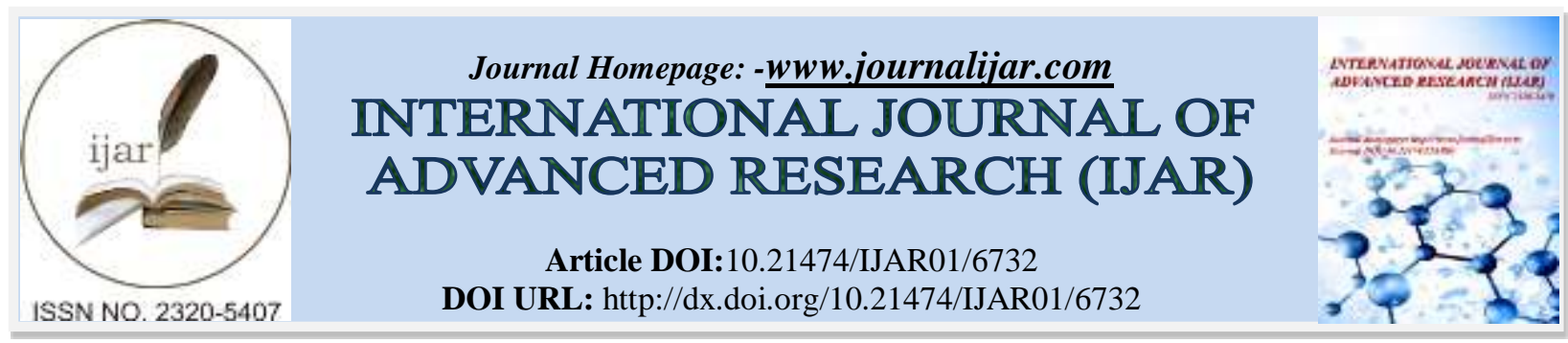

RESEARCH ARTICLE

\title{
IDENTIFICATION AND CLONING OF S-ADENOSYLMETHIONINE DECARBOXYLASE (SAMDC) GENE FROM DICTYOSTELIUM DISCOIDEUM.
}

\author{
Rishikesh Kumar, Priyanka Sharma and Shweta Saran. \\ Jawaharlal Nehru University, New Delhi-110067.
}

\section{Manuscript Info}

Manuscript History

Received: 12 January 2018

Final Accepted: 14 February 2018

Published: March 2018

Keywords:-

Dictyostelium discoideum, Polyamine,

S-adenosylmethionine decarboxylase.

\begin{abstract}
Polyamines are aliphatic polycations involved in cellular processes like growth, differentiation, macromolecular biosynthesis, cell death, cell cycle regulation and embryonic development. Intracellular polyamines pools are maintained by de novo synthesis and transport of polyamines from the extracellular milieu. We have exploited the model organism, Dictyostelium discoideum to understand the role of various polyamines during growth and development. In our earlier publication ${ }^{1}$, we have identified and expressed ornithine decarboxylase (odc) gene from $D$. discoideum and found putrescine to be more important for growth but not development. On the other hand, we also found the importance of spermidine during development but not during growth. Keeping this in view we have presently identified, cloned and expressed the $S$ adenosylmethionine decarboxylase (samdc) gene, which is important for the synthesis of spermidine. We measured the various polyamine levels in isolated prestalk and prespore cells collected from migrating slugs. We have analysed the mRNA expression of samdc gene in the wild type cells, odc overexpressing cells and exogenous putrescine treated wild type cells during development.
\end{abstract}

Copy Right, IJAR, 2018,. All rights reserved.

\section{Introduction:-}

Polyamines (PA) are present in all living cells be it a prokaryote or eukaryote, plants or animals. They are organic cations that are protonated at physiological $\mathrm{pH}$ where, putrescine is divalent, spermidine is trivalent and spermine is tetravalent ${ }^{2}$. All the prokaryotes and the eukaryotes can synthesize putrescine (Put) and spermidine (Spd) but synthesis of spermine $(\mathrm{Spm})$ is restricted to the nucleated eukaryotic cells ${ }^{3,4}$.

Numerous data are available which show that PAs are required for both growth and development however; no agreement is available regarding the mechanisms of involvement of the PAs in these processes. In our previous reports $^{5,6}$, we showed the presence of polyamines during the development of Dictyostelium discoideum. We also showed the involvement of spermidine in development.

D. discoideum, a lower eukaryotic system has both unicellular and multicellular stages in its life cycle. The amoebae grow and divide by feeding on bacteria but upon starvation the solitary amoebae come towards common collecting points in response to cAMP signaling to enter multicellularity. The multicellularity here is not by the repeated division of the "zygote" but by coming together of the spatially segregated cell types. These then undergo various morphogenetic movements to finally culminate into a fruiting body made up of dead vacuolated stalk cells and 
viable spore cells ${ }^{6}$. Cell differentiation during development of Dictyostelium shows a strong correlation to PA levels. We earlier characterized the ornithine decarboxylase (odc) gene from this organism and reported that accumulation of putrescine inhibit cell proliferation but changes in developmental pattern was largely due to effective changes in spermidine and spermine levels. To get a deeper insight into the role of spermidine we have in the present communication identified and expressed the S-adenosylmethionine decarboxylase (samdc) gene, which is involved in the biosynthesis of the polyamines, spermidine and spermine. We also measured the levels of samdc mRNA during development in wild type cells, odc overexpressing cells and after putrescine treatment, which we have earlier reported to increase the spermidine levels ${ }^{1}$.

\section{Materials And Methods:-}

Growth and development of Dictyostelium discoideum:-

D. discoideum Ax2 (axenic strain) cells were grown in HL5 (axenic) medium at $22^{\circ} \mathrm{C}$ under shaken conditions $(200$ $\mathrm{rpm})^{1}$. Nearly after 40 hours of growth, the cells reached a $\log$ phase $\left(\sim 3-5 \times 10^{6}\right.$ cells $\left./ \mathrm{mL}\right)$ after which they were collected and plated for development. Briefly, the cells were harvested and washed in ice-cold $\mathrm{KK}_{2}$ buffer at 2,000 $\mathrm{g}$ for 2 minutes and plated at a density $0.5 \times 10^{6}$ cells $/ \mathrm{mL}$ on non-nutrient agar plates. For synchronization, the plates were incubated at $4{ }^{\circ} \mathrm{C}$ for 6 hours followed by incubation at $22^{\circ} \mathrm{C}$ to allow development progression. The plates were washed with $\mathrm{KK}_{2}$ buffer and samples (different developmental stages) were stored at $-80^{\circ} \mathrm{C}$ to be used later for polyamine isolation.

Development was also carried after treatment with $20 \mathrm{mM}$ putrescine ${ }^{1} .10$ or $20 \mu \mathrm{L}$ drops of cells at a density of $1 \times 10^{7}$ cells/mL were spotted on non-nutrient agar plates ${ }^{1}$.

\section{Identification of SAMDC from $D$. discoideum:-}

Genomic DNA, cDNA and protein sequences of DdSAMDC were obtained from dictyBase online resource (http://www.dictybase.org). Domain architecture of DdSAMDC protein was deduced by Simple Modular Architecture Research Tool (http://SMART.embl-heidelberg.de). SAMDC orthologs were searched using Basic Local Alignment Search Tool (BLASTp) at NCBI (http://blast.ncbi.nlm.nih.gov/Blast.cgi), UniProt and dictyBase. The full length $\mathrm{S}$-adenosylmethionine decarboxylase proenzyme isoform 2 (Homo sapiens) was used to BLAST the D. discoideum genome.

Construction of the overexpressing strain [act15/samdc-eyfp]/Ax2:samdc (DDB_DDB0237590) gene was PCR amplified from Ax2 genomic DNA. The insert (amplicon) and the $\mathrm{pB} 17 \mathrm{~S}$ vector $\mathrm{DNA}^{7}$ were individually double digested with BamHI and XhoI, ligated and the resulting vector was transformed into Ax2 cells by electroporation ${ }^{1}$. The transformants were selected on antibiotic G418 at a maximum concentration of $40 \mu \mathrm{g} / \mathrm{mL}$. The transformants were cloned out on dead bacteria on nonnutrient agar (NNA) plates supplemented with $10 \mu \mathrm{g} / \mathrm{mL}$ G418. The clones were picked up and grown in 1.0 $\mathrm{mL}$ liquid medium and the one with reasonably good fluorescence was selected and stored for further experiments.

\section{Preparation of standard curve for polyamine estimation:-}

To prepare the standard curve with known polyamine levels to measure the unknown values in different samples we quantitated their levels on TLC plates. Mix of known concentrations (ranging from 0-25 $\mu$ moles) of different polyamines was loaded on TLC plates in a fixed volume of $20 \mu \mathrm{L}$. The chromatography was carried on cyclohexane: ethyl acetate $(5: 4, \mathrm{v} / \mathrm{v})$ mix. The plates were dried, visualized and photographed using excitation wavelength of $366 \mathrm{~nm}$ and emission wavelength of $495 \mathrm{~nm}$. The spots developed were measured using the spot densitometry program of Alpha imager. Based on the IDV values given by the software a standard plot was prepared. This helped us to draw a standard curve for the different polyamines. Whenever an unknown sample was run, a standard mix was also run and the values, which were observed, helped us quantitate the unknown polyamine levels. In order to keep the parameters more or less constant, we also captured the photograph of the gel plate under same parameters.

\section{Polyamine estimation:-}

Polyamines were extracted either from growing cultures or specific developmental stages according to the method described by ${ }^{1}$. Briefly, cells ( $2 \times 10^{8}$ cells) were washed with $\mathrm{KK}_{2}$ buffer and $200 \mu \mathrm{L}$ of $10 \%$ perchloric acid (v/v) was added to the pellet and mixed. $100 \mu \mathrm{L}$ of this suspension was taken for polyamine extraction and the rest was 
used for protein estimation by Bradford ${ }^{8}$ method. The mixture was kept at $4^{\circ} \mathrm{C}$ for 1.0 hour after which $100 \mu \mathrm{L}$ of $1 \mathrm{~N} \mathrm{NaOH}$ was added. The samples were hydrolysed with $12 \mathrm{~N} \mathrm{HCl}(1: 1 \mathrm{v} / \mathrm{v})$ for $16-18$ hours at $110^{\circ} \mathrm{C}$ in flame sealed glass ampoules. Hydrolysates were filtered through glass wool and washed with 6 drops of $6 \mathrm{~N} \mathrm{HCl}$. It was dried at $70^{\circ} \mathrm{C}$ on dry bath in a hood and then resuspended in $50 \mu \mathrm{L}$ of $10 \%$ perchloric acid.

$50 \mu \mathrm{L}$ of both standard and extracted polyamine mix were mixed with $50 \mu \mathrm{L}$ of water saturated $\mathrm{Na}_{2} \mathrm{CO}_{3}$. To this 10 $\mu \mathrm{L}$ of $100 \mathrm{mg} / \mathrm{mL}$ acetone of dansyl chloride was added. The mixture was vigorously mixed in a vortex and incubated in dark at $26^{\circ} \mathrm{C}$ overnight. Excess dansyl chloride was removed by the addition of $5 \mu \mathrm{L}$ of proline $(1 \mathrm{~g} / \mathrm{mL}$ of water) and incubated in dark for 30 minutes. The mixture was then extracted with $100 \mu \mathrm{L}$ of benzene and the organic phase was collected. The sample thus prepared was loaded as $20 \mu \mathrm{L}$ spots on the TLC plates. The chromatogram was developed as earlier and the spots developed were measured. The values were obtained using the standard curve prepared from different concentrations of the mix. This was finally calculated on the basis of protein content of the sample.

\section{Isolation of prestalk and prespore cell population:-}

The Ax2 cells were synchronously developed till the migrating slug stage. Tubes containing $50 \%$ percoll were centrifuged for 30 minutes at $14,000 \mathrm{~g}$ at $4^{\circ} \mathrm{C}$ to create a linear gradient. Meanwhile, the slugs were collected onto a $77 \mu \mathrm{m}$ mesh that allowed single cells to pass through. Slow centrifugation for 1 minute at $500 \mathrm{~g}$ at $4^{\circ} \mathrm{C}$ allowed the slugs to pellet down. The slugs were then treated with cold pronase-dissociation solution to dissociate into single cells with repeated purging with a Pasteur pipette up to 10 minutes and the dissociation was confirmed by microscopic examination. The cells were washed twice with cold $1 \times \mathrm{KK}_{2}$ buffer containing $20 \mathrm{mM}$ EDTA at 2, $000 \mathrm{~g}$ for 5 minutes at $4^{\circ} \mathrm{C}$. The cell suspension was loaded slowly and equally on the percoll gradient tubes on ice and spun at $15,000 \mathrm{~g}$ for 3 minutes at $4^{\circ} \mathrm{C}$. The prestalk and prespore cells made separate density dependent layers. The prestalk cells being lighter made an upper layer, while prespore cells made a lower layer of cells. The cells were separately collected and washed in cold $1 \times \mathrm{KK}_{2}$ buffer with $20 \mathrm{mM}$ EDTA to remove the percoll completely. The differentiating cell types, thus collected, were stored at $-80^{\circ} \mathrm{C}$ until used ${ }^{9}$. The purity was checked using any cell type specific marker or by neutral red dye. The preparation was found to be more than $90 \%$ pure.

\section{Results And Discussion:-}

Identification and cloning of samdc gene from Dictyostelium discoideum:-

The full length S-adenosylmethionine decarboxylase proenzyme isoform 2 [Homo sapiens] was used as query to BLAST the D. discoideum genome. The DdSAMDC (DDB0237590) shares 45\% identity with the human SAMDC. Full-length sequences from human and $D$. discoideum putative SAMDC proteins were multiple aligned using ClustalW2 at EBI server (Fig. 1A). Ddsamdc gene with an id DDB0237590 (having an E value of 1e-65; score bits 246) is located on chromosome 2 between coordinates 5517792 to 5519439 on the Watson strand. Ddsamdc is a single copy gene having 379 amino acids and a calculated mass of $42.57 \mathrm{kDa}$. Domain architecture analyses as predicted by the SMART program shows the presence of a SAM decarboxylase domain (26-352 amino acids) (Fig. 1B). Fig. 1C shows the multiple sequence alignment of the SAMDC domain of both human (Hs) and Dictyostelium $(D d)$. This enzyme catalyses the removal of the carboxylate group of SAM to form $\mathrm{S}$ adenosyl 5'-3-methylpropylamine which then acts as the n-propylamine group donor in the synthesis of the polyamines spermidine and spermine ${ }^{10}$. 


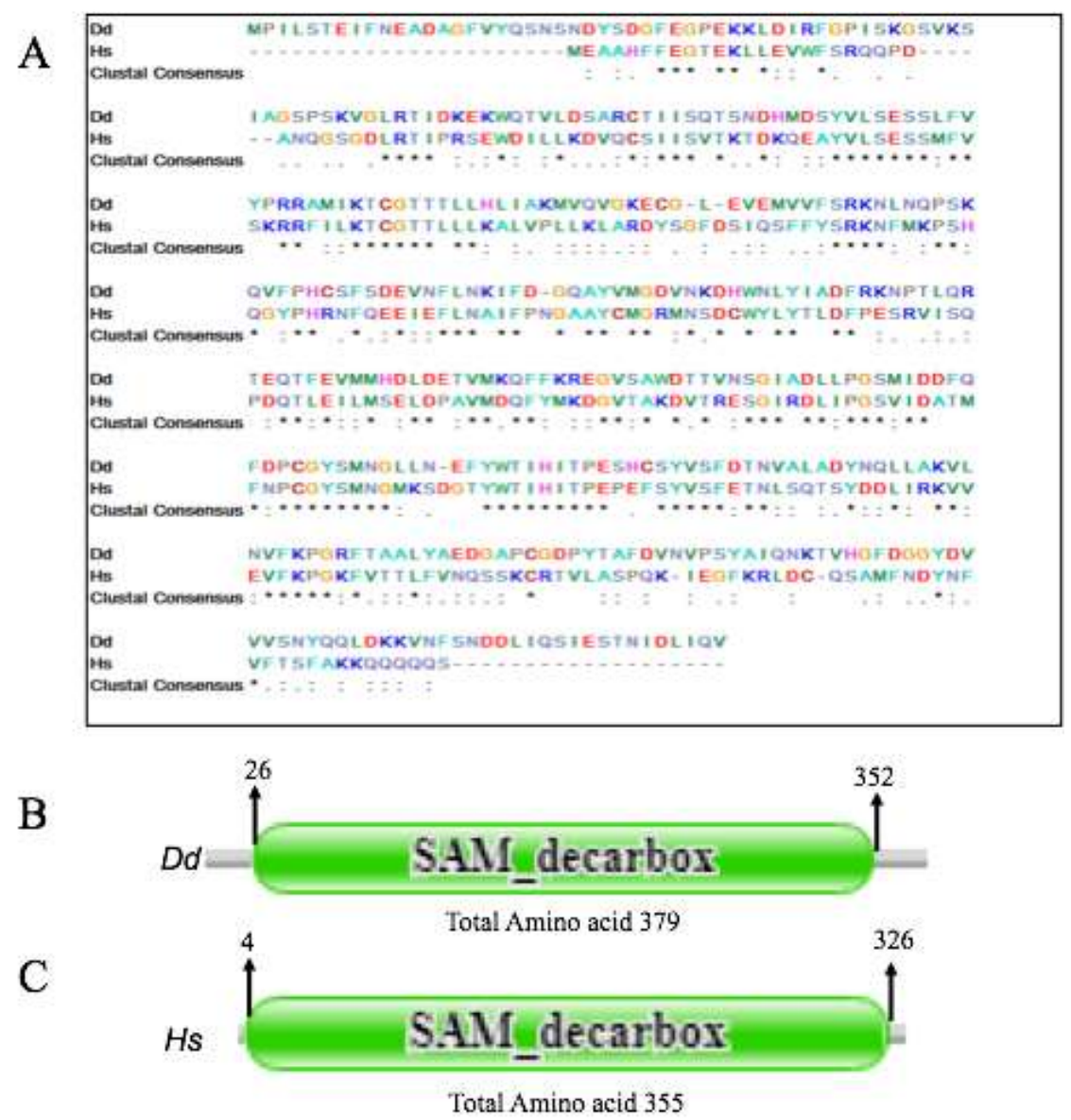

Fig. 1: The multiple sequence alignment of SAMDCs. (A) Multiple sequence alignment of SAMDC domains of Dictyostelium discoideum (DDB0237590) and human (NP_001625.2) using ClustalW2. It shows 45\% identity. The SAMDC domain as predicted by the SMART programme for (B) D. discoideum (26-352 amino acids) and (C) Homo sapiens (4-326 amino acids).

\section{Cloning of samdc gene from $D$. discoideum:-}

The putative Ddsamdc was expressed under the constitutively active actin15 promoter as a fusion protein with eYFP (a reporter gene) at the C-terminal (Fig. 2A). The following primer pair having the restriction sites (underlined) was used for the amplification.

Forward: (BamHI): 5'- CTGAGGATCCCCAATTTTGTAAGGCCAA -3'

Reverse: (XhoI): 5'- CTGACTCGAGAAATATTAGTTGATTCGATTGATT -3'

The 1.1 Kb size amplicon was PCR amplified (Fig. 2B), double digested with BamHI/XhoI and ligated to the BamHI/XhoI digested $\mathrm{p}($ acg-eYFP $)$ vector ${ }^{7}$. The vector construct was checked with appropriate digestions like BamHI/XhoI, BamHI/XbaI and BamHI only, which gave the expected bands. The fall out of $1.1 \mathrm{~Kb}$ with BamHI/XhoI and a $6.1 \mathrm{~Kb}$ vector backbone was observed. The BamHI/XbaI gave the $1.8 \mathrm{~Kb}$ fall out (amplicon plus $700 \mathrm{bp}$ size of eyfp) and the vector backbone of $5.33 \mathrm{~Kb}$ sizes. BamHI yields a linearized vector of 7.2 $\mathrm{Kb}$ size (Fig. 2C). The construct was further confirmed by sequencing (data not shown) and transformed by electroporation ${ }^{1}$ into Ax2 cells to obtain the overexpressing strain, act15(samdc-eyfp)/Ax2.

The observations confirmed that eYFP (green) tagged $D$. discoideum SAMDC is localized in the cytoplasm (Fig. 2D). The predominant presence of SAMDC-eYFP in the cytosol is in agreement with the vast majority of earlier studies, which showed cytoplasmic localization ${ }^{11}$. The role of the SAMDC/polyamine system in the cytoplasm still needs to be elucidated, but numerous studies suggest that polyamines play essential roles in the machinery of protein biosynthesis. 

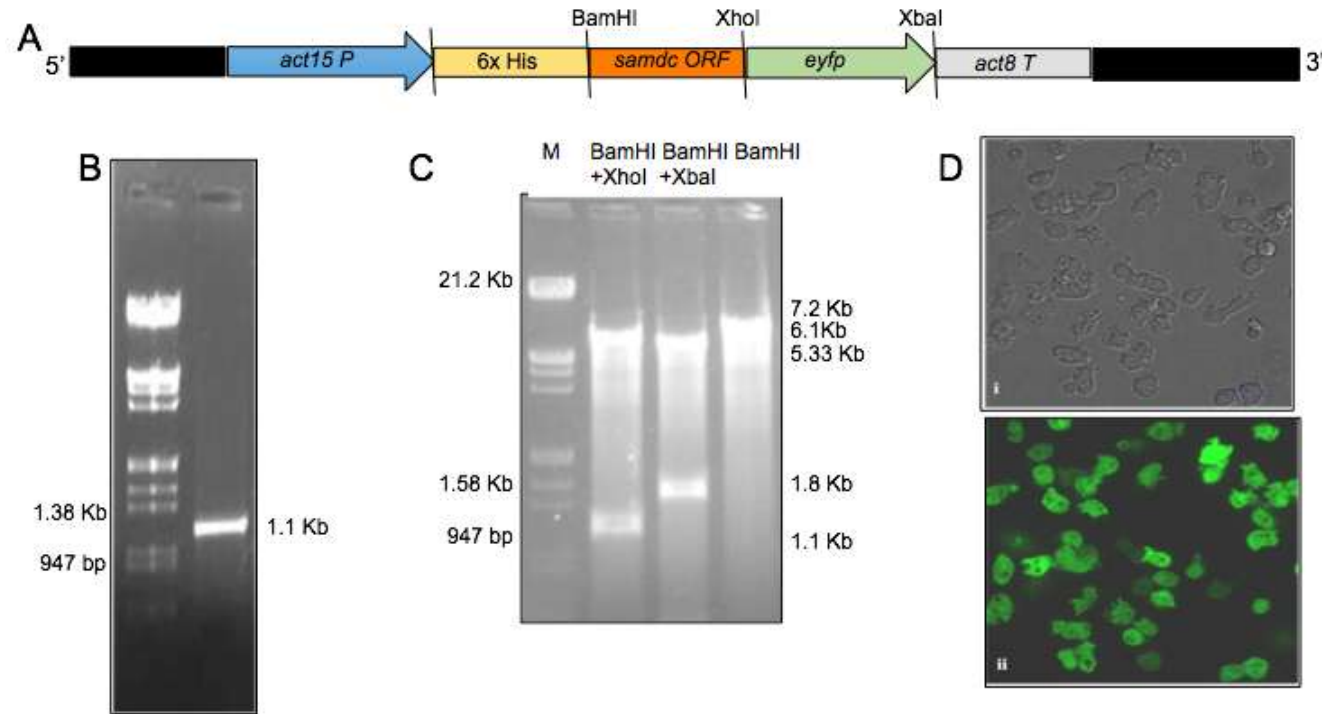

Fig. 2: PCR amplification, cloning and expression of Ddsamdc (DDB0237590 sequence ID) gene. (A) Cartoon showing the construct. (B) Genomic region was amplified as a $1.1 \mathrm{~Kb}$ fragment. (C) The vector was digested with the restriction enzymes BamHI/XhoI to release the $1.1 \mathrm{~Kb}$ insert and a $6.1 \mathrm{~Kb}$ vector backbone. Digestion with $\mathrm{XbaI} / \mathrm{BamHI}$ yield fragments of $1.8 \mathrm{~Kb}$ and $5.33 \mathrm{~Kb}$ sizes. The BamHI digestion linearized the vector to give a $7.2 \mathrm{~Kb}$ fragment. (The actual sizes of the DNA fragments are marked; M- DNA marker). (D) Subcellular localization studies in constitutively expressing [act15/samdc-eyfp]/Ax2 cells. Upper: DdSAMDC-eYFP DIC image; lower: eYFP fluorescence of DdSAMDC-eYFP cells.

Preparation of standard curve for polyamine estimation in isolated prestalk and prespore cells:-

Here we have measured the levels of various polyamines, namely putrescine, spermidine and spermine in isolated prestalk and prespore cells collected from migrating slugs. Fig. 3A shows the image of one representative TLC plate with varying concentrations (0.2-20 $\mu$ moles) of different polyamines loaded for the preparation of a standard curve. Based on the intensity of the spots developed a standard curve for the different polyamines were prepared. The photographic images of different TLC plates were always taken at the same specifications to rule out any variations if any and also the standard sample mix were loaded on every gel when unknown samples were to be quantitated. Fig. 3B-D represents the standard curves for putrescine, spermidine and spermine, respectively and the values obtained were as $0.025 \mathrm{nmoles} / \mathrm{IDV} / 20 \mu \mathrm{l}, 0.019 \mathrm{nmoles} / \mathrm{IDV} / 20 \mu \mathrm{l}$ and $0.021 \mathrm{nmoles} / \mathrm{IDV} / 20 \mu \mathrm{l}$.

A
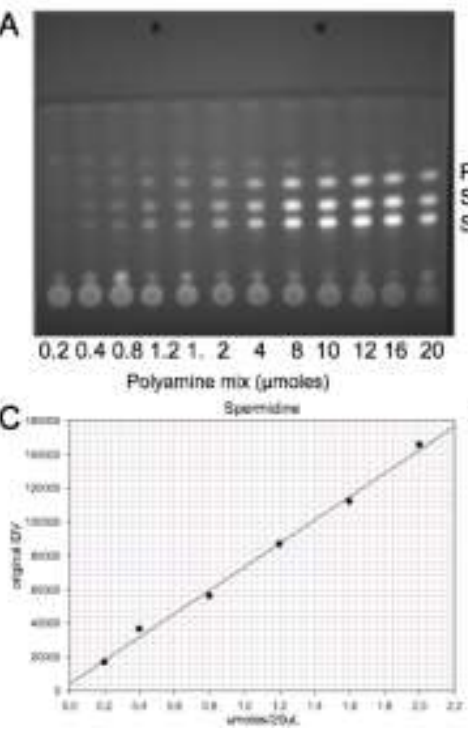

B

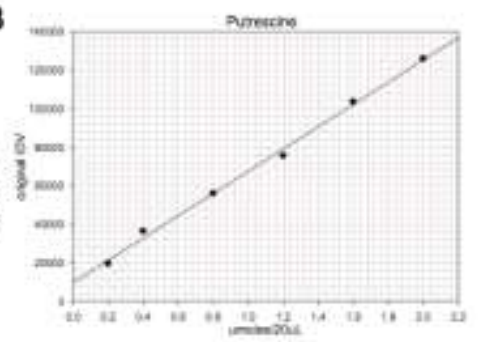

D

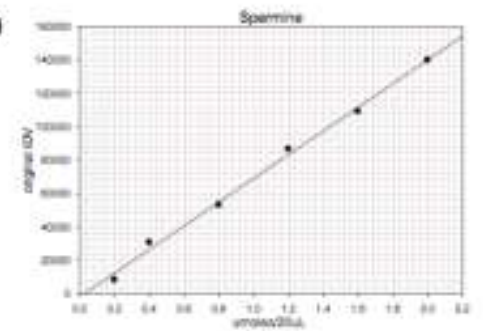


Fig. 3: Standard curve for various polyamines using known concentrations of individual polyamines. (A) An image of one such TLC plate where varying concentrations of the standard polyamine mix was loaded. The concentration of each polyamine is marked. (B) Standard plot for putrescine where 1 IDV value $=0.025$ nmoles. $(C)$ Standard plot for spermidine where 1 IDV value $=0.0194$ nmoles. (D) Standard plot for spermine where 1 IDV value $=0.021 \mathrm{nmoles}$. [n=6].

Since, polyamines play a role in differentiation, we next wanted to measure their levels in isolated prestalk and prespore cells derived from migrating slugs (Fig. 4). Prestalk and prespore cells are precursors of the terminally differentiated stalk and spore cells. This stage is important as the cells have not yet entered terminal differentiation but are just committed to the developmental pathways. As shown, the levels of all the three polyamines are higher in the isolated prespore cells as compared to the isolated prestalk cells. There was nearly two-fold increase in case of putrescine and spermidine but we did not find any significant increase in the levels of spermine. The above observations suggest the following: i) all the three polyamines are required for the process of differentiation but may be in varying relative ratios. ii) Putrescine is required in larger amounts for the synthesis of its downstream products like spermidine and spermine. It was also observed, that prestalk cells which are destined to die, requires less amounts of polyamines as compared to prespore cells that are to remain viable.

A

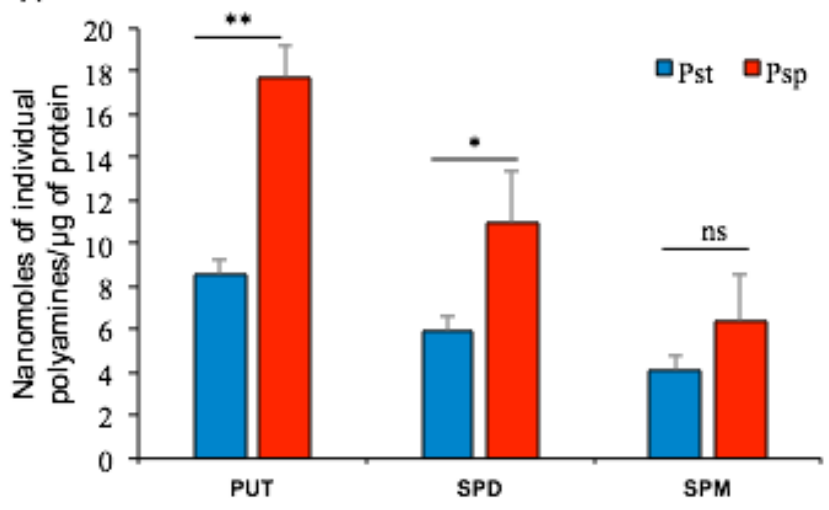

Fig. 4: Polyamine levels in isolated prestalk and prespore cells. Cells from migrating slugs were collected and subjected to polyamine estimations. Levels of putrescine (put); spermidine (spd) and spermine (spm) were measured and the values are expressed as nanomoles/ $\mu \mathrm{g}$ of protein. $[\mathrm{n}=6]$ [pst-prestalk; pspprespore].

Ddsamdc transcript pattern during development of $D$. discoideum;-

We next wanted to find the samdc mRNA levels during development of wild type cells and compare them to that observed when the spermidine levels were increased due to overexpression of samdc or by putrescine treatment ${ }^{1}$ (Fig. 5). The mRNA levels during the development of wild type cells increased slightly but showed an abrupt increase during culmination suggesting spermidine to be important for terminal differentiation (Fig. 5A). In the constitutively expressed samdc cells where the level of spermidine is supposed to be high, we did not observe increased expression of samdc mRNA levels. In fact, the levels were low throughout development as compared to the wild type suggesting that overexpression increased the effective spermidine levels that may be required for the terminal differentiation of cells (Fig. 5B). When the wild type cells were treated with putrescine there was an effective increase in the spermidine levels also ${ }^{1}$. We had earlier observed largely increased putrescine levels and small increase in spermidine and spermine levels upon treatment with putrescine. Thus, in this case we observed a small increase in samdc mRNA expression as compared to the samdc overexpressing cells but much less than that observed in wild type cells. 

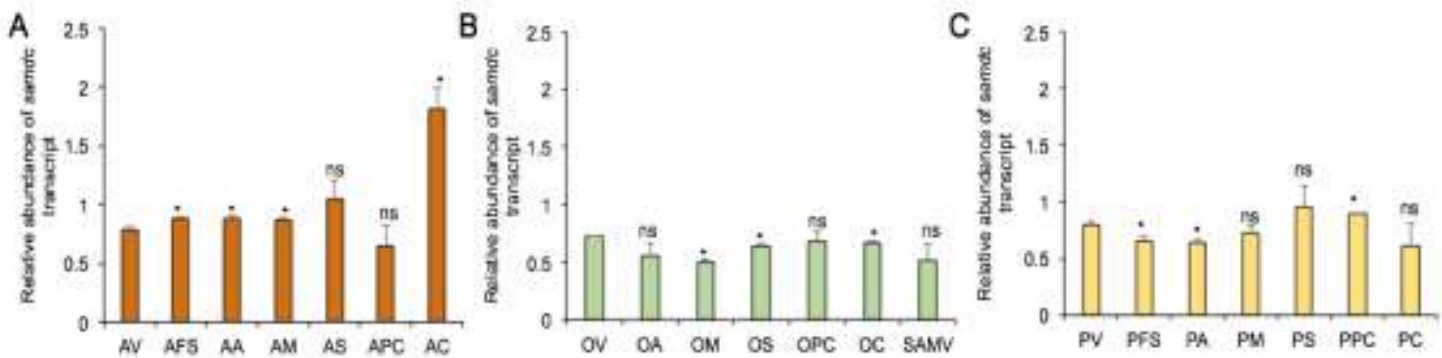

Fig. 5: Ddsamdc transcript pattern during development. (A) samdc expression during different developmental stages developed from wild type Ax2 cells. (B) samdc expression during different developmental stages in odc-eyfp overexpressing cells. (C) samdc expression in Ax2 cells treated with 20mM putrescine. [A: A stands for Ax2 cells; B: O stands for odc overexpression and C: $\mathrm{P}$ stands for putrescine treatment. In all V: vegetative; FS: freshly starved; A: aggregation; M: mound; EC: early culminant; C: culminant; $n=4]$.

Previous literatures have already shown that high polyamine levels are toxic to the cell survival so there could be transporters present to maintain homeostasis. To maintain the optimum levels of polyamines during the specific stages of development Dictyostelium discoideum has adopted different mechanism. Lot more studies are still awaited to answer these questions.

\section{Conclusions:-}

$S$-Adenosylmethionine decarboxylase (SAMDC, EC 4.1.4.50) is a rate-limiting enzyme in the biosynthesis of polyamines from putrescine ${ }^{12}$. The $s a m d c$ gene is widespread in nature and occurs from fungus to yeast. SAMDC helps in the synthesis of spermidine, which has diverse roles to play in various biological functions. SAMDC is one of the key enzymes in polyamine metabolism, which converts $\mathrm{S}$-adenosylmethionine (SAM) into decarboxylated SAM, which serves as an aminopropyl donor to convert putrescine and spermidine into spermidine and spermine, respectively.

To modulate the levels of spermidine we overexpressed the samdc gene or treated the cells with exogenous putrescine. This led to direct increase in spermidine levels and subsequently other polyamines also ${ }^{13,14}$. Here we report the isolation of a putative clone of the samdc overexpressing cells.

Presently, we have measured the levels of different polyamines, namely putrescine, spermidine and spermine in isolated prestalk and prespore cells collected from migrating slugs. In order to evaluate the value of the individual polyamines we successfully created a standard plot for each of the polyamine.

The polyamines are ubiquitously present across the species and are known physiological cations. They are essential for normal cell growth and differentiation but their function at the molecular level is still not understood. They probably bind to the various negatively charged structures and macromolecules in cells, the most important being DNA. We show that $D$. discoideum which is like a prokaryote (when unicellular) and eukaryote (when multicellular) has all the three polyamines. At different stages of development, the level of polyamines determines the progression through to the next stage of development. We propose that several intracellular threshold levels of different polyamines exist.

\section{Statistical analysis:-}

The statistical analyses were performed (mean standard deviation and standard error) and values were plotted in graph using Microsoft EXCEL-2013. p-Values of less than 0.05 were considered as significant.

\section{Data accessibility:-}

The datasets supporting this article is present here.

\section{Author contributions:-}

S.S. and R.K. conceived and designed the experiments. R.K. and P.S. performed the experiments. S.S. and P.S. wrote the manuscript. 


\section{Competing interests:-}

We declare no conflict of interest.

\section{Funding:-}

Funding from UGC [\# 42-181/2013(SR)] to S.S. is acknowledged. Institutional fundings in the form of DSTPURSE, FIST-II, UGC-DRSI is acknowledged. R.K. and P.S. thank the UGC for research fellowships.

\section{References:-}

1. Kumar R, Rafia S, Saran S. Cloning, expression and characterization of the ornithine decarboxylase gene from Dictyostelium discoideum. Int J Dev Biol. 2014; 58(9): 669-76.

2. Wallace HM. Polyamines: specific metabolic regulators or multifunctional cations? Biochem Soc Trans. 1998; 26:569-71.

3. Marton LJ, Pegg AE. Polyamines as targets for therapeutic intervention. Annu Rev Pharmacol Toxicol. 1995; 35:55-91.

4. Thomas T, Thomas TJ. Polyamines in cell growth and cell death: molecular mechanisms and therapeutic applications. Cell Mol Life Sci. 2001; 58(2): 244-58.

5. Saran S. Changes in endogenous polyamine levels are associated with differentiation in Dictyostelium discoideum. Cell Biol Int. 1998; 22(7-8):575-80.

6. Schaap P. Evolution of developmental signalling in Dictyostelid social amoebas. Curr Opin Genet Dev. 2016; 39:29-34.

7. Saran S, Schaap P. Adenylyl cyclase G is activated by an intramolecular osmosensor. Mol Biol Cell. 2004; 15(3):1479-86.

8. Kruger NJ. The Bradford method for protein quantitation. Methods Mol Biol. 1994; 32:9-15.

9. Gosain A, Srivastava A, Saran S. Peptide: N- glycanase is expressed in prestalk cells and plays a role in the differentiation of prespore cells during development of Dictyostelium discoideum. Indian J Exp Biol. 2014; 52(3):197-206.

10. Tiburcio AF, Altabella T, Borrel A, Masgrau C. Polyamine metabolism and its regulation. Physiol Plant. 1997; 100:664-674.

11. Schipper, RG, Verhofstad, AA. Distribution patterns of ornithine decarboxylase in cells and tissues: facts, problems, and postulates. J Histochem Cytochem. 2002; 50(9): 1143-60.

12. Gilmour SK. Polyamines and non-melanoma skin cancer. Toxicol Appl Pharmacol. 2007; 224(3): 249-56.

13. Tabor CW, Tabor H. Polyamines. Annu Rev Biochem. 1984; 53:749-90.

14. Pegg AE. Recent advances in the biochemistry of polyamines in eukaryotes. Biochem J. 1986; 234(2): 249-62. 